\title{
Profile of darunavir in the treatment of HIV-infected pediatric and adolescent patients
}

This article was published in the following Dove Press journal:

Adolescent Health, Medicine and Therapeutics

I3 September 201 I

Number of times this article has been viewed

\section{Joanna Dobroszycki \\ Jacobo Abadi \\ Andrew A Wiznia \\ Michael G Rosenberg}

Department of Pediatrics, Division of Infectious Diseases, Jacobi Medical Center, Bronx, NY, USA
Correspondence: Michael G Rosenberg Jacobi Medical Center, 1400

Pelham Parkway So, IW-5,

Bronx, NY 1046I, USA

$\mathrm{Tel}+\mathrm{I} 7189184677$

Fax +17189184699

Email michael.rosenberg@nbhn.net

\begin{abstract}
The introduction of protease inhibitors (PI) containing antiretroviral regimens in the treatment of HIV infection in infants, children, and adolescents has dramatically decreased morbidity and mortality. Darunavir, the latest PI to be FDA approved for pediatric patients older than 6 years and currently the preferred PI for use in adult patients, was added as an alternative PI for use in children based on a combination of data from both adult and pediatric trials. This review of darunavir in the treatment of HIV-infected children and adolescents looks at the major published clinical trials findings, pharmacokinetic and resistance studies, and preliminary data on use in younger children.
\end{abstract}

Keywords: darunavir, HIV, protease inhibitor

\section{Introduction}

The introduction of protease inhibitors (PI) containing antiretroviral (ARV) regimens in the treatment of HIV infection in infants, children, and adolescents has dramatically decreased morbidity and mortality. ${ }^{1,2}$ At the same time, increased survival rates and longevity have given rise to new challenges as patients and caregivers deal with adherence issues, long-term drug toxicities, "treatment fatigue," and difficulties in transitioning to adulthood and adult care settings. ${ }^{3,4}$ Historically, progress in pediatric ARV drug development and licensure has lagged behind advances in the treatment of adult HIV infection. Pediatric pharmacology is challenging on many levels, ranging from difficulties in making palatable liquid/powder formulations for infants and young children, the need to adjust for metabolic changes associated with organ growth and puberty, to the limited availability of pediatric subjects for enrollment in large-scale clinical trials. ${ }^{5,6}$

Children and adolescents are not miniature adults: a lesson learned early in the course of pediatric antiretroviral drug development ${ }^{7}$ and well illustrated by the nelfinavir pharmacokinetics (PK) experience of Pediatrics AIDS Clinical Trial Group Protocol 377. That trial demonstrated that children require a much higher dose of nelfinavir, based on body weight, than adults, to maintain acceptable drug levels, a finding that was not adequately addressed in the US Food and Drug Administration (FDA) approved package insert. ${ }^{8}$

The 2010 edition of the Guidelines for the Use of Antiretroviral Agents in Pediatric HIV infection includes recommendations based on findings from randomized clinical trials in adults combined with data from nonrandomized pediatric trials demonstrating adequate and consistent safety and PK results in children. ${ }^{9}$ Currently the preferred PI 
regimen for initial therapy in children is low-dose ritonavir (RTV)-boosted lopinavir (LPV/r) in combination with two nucleoside reverse transcriptase inhibitors (NRTIs). Low-dose RTV-boosted atazanavir and fos-amprenavir are alternative PI-based regimens. ${ }^{9}$ Ritonavir-boosted darunavir (DRV/r), the latest PI to be FDA approved for pediatric patients $>6$ years old (December 2008, just 2 months after adult approval), and currently the preferred PI for use in adult patients, was added in 2010 as an alternative PI for use in children based on a combination of data from both adult and pediatric clinical trials, the POWER,${ }^{10}$ ARTEMIS,${ }^{11}$ and the DELPHI $^{12}$ trials.

The reasons, at present, for DRV/r not being the preferred initial therapy PI for children include: (1) limited pediatric experience compared with RTV-boosted lopinavir (LPV/r), the current preferred PI; (2) lack of pediatric studies in treatment-naïve patients; (3) lack of a liquid formulation and the high pill burden with the $75 \mathrm{mg} \mathrm{DRV} / \mathrm{r}$ pediatric tablet formulation. Importantly, DRV/r is approved only for twice daily dosing in children and does not have an approved indication for children $<6$ years of age. ${ }^{9}$

In this review, we will describe and discuss the use of $\mathrm{DRV} / \mathrm{r}$ in the treatment of HIV-infected children and adolescents. We will look at the published trials data, including PK and resistance studies, as well as preliminary data on liquid formulations, DRV/r use in younger patients, and once daily dosing.

\section{Pediatric ARV management: general considerations}

In general, the approach clinicians adopt when making therapeutic choices balances the potential effectiveness and overall benefits of the regimen versus the challenges that the treatment carries. When deciding to start or change ARVs in HIV-infected children or adolescents one must not only consider the above mentioned factors, but also which ARVs have available pediatric formulations, have proven PK profiles, and whether the treatment offered is appropriate to the child/adolescent's developmental and psychosocial situation. Caregiver assessment and education is also of crucial importance. In other words, the question to consider is whether or not the patient will adhere and benefit from the regimen without compromising future options. ${ }^{13}$

Thirty years into the HIV epidemic, the expectation for new pharmacologic agents is that they not only be potent, effective, and well tolerated, but also that they have the safest profile with minimal complications. However, unknown and/or long-term side effects often cannot be judged because we simply do not have the longitudinal experience to make these assessments. This is particularly true for pediatric patients because of the very young age at which treatment is initiated. In addition, we know that the responses to combination antiretroviral therapy (cART) are variable and that immune recovery is never complete despite sustained and acceptable improvements and stability of immunologic and virologic parameters. ${ }^{14}$

The HIV-infected pediatric and adolescent patients pose unique management challenges. The perinatally infected adolescents and young adults who live predominantly in resource-rich countries (US and countries of Western Europe), have been exposed to sequential courses of therapy and, once reaching adolescence and young adulthood, have typically accumulated significant drug-resistant viruses which can be particularly difficult to control. Adolescents who have been infected through high-risk behaviors may also harbor acquired resistant strains or could have developed resistance themselves depending on the length of the infection, its progression, ARV exposure, and adherence patterns. Younger children, who are more likely to be vertically infected, may have acquired resistant strains from their mother or developed resistance depending on the available cART, adherence to effective treatment, and the time for follow-up to detect virologic failure associated with drug resistance. Thus, drug development to address these challenges can be a daunting task.

\section{Clinical efficacy, tolerability and adverse effects of darunavir as shown by clinical trial data}

Darunavir is a high affinity nonpeptidic HIV-1/2 protease inhibitor, which has demonstrated activity against wild type and most drug-resistant HIV, and it is usually co-administered with other active ARVs. Bioavailability is enhanced with low-dose RTV as the so called boost. ${ }^{15}$

The first published pediatric trial of DRV/r was the DELPHI study, which looked at the PK, efficacy, and safety of DRV/r in treatment-experienced children and adolescents. ${ }^{12}$ This international, multicenter open label phase II study enrolled 80 HIV-infected children/adolescents between the ages of 6 and 17 years. Patients had viral loads (VL) $>1000$ copies $/ \mathrm{mL}$ (median $4.64 \log _{10} \mathrm{cop} / \mathrm{mL}$ ) and stable CD4 + T lymphocyte counts (median 330 cells $/ \mu \mathrm{L}$ ). Patients in the study tended to be older and heavily treatment-experienced. The median age was 14 years $(70 \%>12$ years $)$; the median duration of HIV infection was 11 years; and median duration of ARV therapy 115.5 months, with median number of nine previous 
ARV (all patients had at least three: $100 \% \geq 2$ NRTIs, $79 \%$ NNRTI, and $96 \%$ exposed to $\geq 1 \mathrm{PI}$ ). All patients received weight-based DRV/r dosed twice daily, plus an optimized background regimen consisting of at least two NRTIs (98\%), non-nucleoside reverse transcriptase inhibitors (NNRTIs) $(6 \%)$, or the fusion inhibitor enfuvirtide $(30 \%)$.

The primary end point was $\geq 1 \log _{10}$ reduction in VL with data analysis performed at 24 and 48 weeks.

The results indicated that $74 \%$ and $65 \%$ of the cohort achieved $\geq 1 \log _{10}$ VL reduction at 24 and 48 weeks, respectively. At 48 weeks, $59 \%$ and $48 \%$ of the patients had VL $<$ S400 and $<50$ copies $/ \mathrm{mL}$, respectively. Virologic response was sustained, with better response rates in children between 6 and 12 years of age, probably due to higher baseline levels of resistance related to greater treatment experience in the older 12- to 17-year age group. Logistic regression analysis identified two significant variables associated with VL $<50$ copies/mL at 48 weeks: adherence and baseline DRV/r resistance-associated mutations. There was a median increase of T cells at 48 weeks of 110 cells $/ \mu \mathrm{L}$. In addition, at the end of the study, age and sex-adjusted weight and height $z$-scores showed significant changes compared with baseline measurements.

Overall, DRV/r was well tolerated with most adverse events reported as mild to moderate. Grades 2-4 clinical events were reported in 2/80 (grade 2 diarrhea, grade 2 rash). These resolved without sequelae and did not require DRV/r dose adjustment, treatment interruption, or discontinuation. Furthermore, most laboratory abnormalities were grades $1-2$. Values from baseline were not considered clinically relevant and event grades 3-4 were recorded in $26 \%$ of the patients. Of the children who had elevated triglycerides at baseline (30/80), the majority had been previously exposed to LPV/r, and over time there was a trend for lower triglyceride levels. Only one patient discontinued DRV/r due to a pre-existing condition. There were no deaths reported. The results of the DELPHI study were pivotal for the FDA approval (December 2008) of DRV/r use in patients $>6$ years of age. ${ }^{15}$

In attempting to identify predictors of success versus failure in treatment-experienced pediatric patients, one may need to look for guidance at the larger adult studies which have addressed these questions: The POWER trials, addressing DRV/r use in highly experienced patients, and the TITAN study conducted in treatment-experienced patients but naïve to LPV/r. The conclusions from these studies suggest that optimal response to DRV/r depends on the quality and quantity of baseline RAMs, and the potency of other active drugs used in combination with DRV/r. The former is likely related to previous ARV experience, and of note is the fact that prior failure with the PIs amprenavir or fos-amprenavir may have a negative impact on DRV/r efficacy, likely related to their structural similarities. In contrast, previous exposure to the PI tipranavir (TPV) appears not to have a significant effect on DRV/r success. ${ }^{10,16,17}$

A recent report from France studied twelve perinatally HIV-infected adolescents with virological failure and documented triple class multidrug resistant virus. ${ }^{18}$ All patients were started on a regimen containing DRV/r, etravirine (newgeneration NNRTI), and raltegravir (integrase inhibitor). In eight cases, one or several additional drugs were added to the backbone triple combination, including one or two NRTIs in seven cases, tenofovir in four and enfuvirtide in three. At baseline, the cohort had a median age of 15 years, median weight of $48 \mathrm{~kg}$, median CD4 of 124 cells $/ \mu \mathrm{L}$, and median VL of $5.3 \log _{10}$ copies $/ \mathrm{mL}$. They all had been exposed to at least one NNRTI, and the median exposures to NRTIs and PIs were six and four, respectively. Eight patients had CDC Class C. After 12 months, VL had dropped to a median of $1.6 \log _{10}$ cop/mL, and CD4 had increased to a median of 500 cells/ uL. Only one patient had to discontinue DRV because of gastrointestinal side effects. No other grade 3-4 adverse events, clinical or laboratory, were reported.

Finally, there has been a series of studies involving adult cohorts addressing the use of once daily DRV/r: (1) in experienced patients but without baseline DRV/r mutations, (2) comparing to LPV/r QD in treatment-naïve patients, (3) by itself or with two nucleoside analogues in patients with sustained viral suppression. ${ }^{19-21}$ The results and conclusions from the cited studies provide supporting evidence for the use of DRV/r on the basis of efficacy, tolerability and improved metabolic profile compared to LPV/r. Moreover, there are validated predictors reported in a recent study of a highly antiretroviral-experienced adult cohort to help guide the decision to use DRV/r in a new cART regimen, including but not limited to the quality and quantity of baseline major PI mutations, previous exposure to TPV, current first use of enfuvirtide, and current use of raltegravir. ${ }^{22}$

These results support DRV/r use as an effective and well-tolerated alternative in highly treatment-experienced children and adolescents. It is in this group where currently most efforts are concentrated to monitor the long-term efficacy, and development of treatment-related complications. As will be discussed in greater detail below, ongoing trials are evaluating safety and efficacy of twice daily DRV/r in treatment-experienced HIV-infected children 3-6 years of age with weights $>10 \mathrm{~kg}$ but $<20 \mathrm{~kg}$, and once daily DRV/r 
in treatment-naïve adolescents $>12$ and $<18$ years of age weighing $>40 \mathrm{~kg}$.

\section{Pharmacokinetics: theory and practice \\ Theoretical considerations}

As noted already, clinical development and regulatory approval of antiretrovirals for use in pediatric populations is tightly dependent upon the results from clinical trials conducted in adult populations. In adults, successful phase III clinical trials demonstrate efficacy and safety of a novel agent and result in a "chosen" dose, which, if and when approved by regulatory agencies such as the FDA and the European Medicines Agency, becomes the commercially available product. Concurrent, or subsequent, adult studies better define PK drug-drug interactions which may have significant impact on both efficacy and safety. Early results from these adult phase II and III trials are utilized to plan and conduct clinical trials in pediatric populations. The primary objectives of most initial ARV trials in pediatrics include studying PK and short- and long-term toxicities with the goal of choosing the correct and safe dose of the agent. Efficacy and other topics of interest are typically reserved as secondary objectives in these trials. The assumption in this process is that the PK parameters obtained with a treatment dose that has demonstrated safety and efficacy in adults can serve as a surrogate marker for the development and modeling of pediatric dosing as similar drug exposure in children should demonstrate equivalent efficacy and safety.

This task of choosing a dose in children, which appears to be relatively simple, is actually quite challenging as pediatric cohorts are diverse and include newborns, infants, children, adolescents, and young adults, and each one may have unique PK variables that may affect drug distribution and metabolism. Typically, HIV-infected newborns metabolize drugs more slowly than adults but this rapidly transitions into a state where young children metabolize these agents much more rapidly than adults and, if not fully defined in carefully constructed trials, a presumed appropriate dose may adversely affect the tolerability of the agent for that individual. The need to develop appropriate pediatric drug preparations is another complicating factor. Liquid preparations are problematic due to concerns about stability and storage across a range of temperatures as well as volume and palatability. When tablets and capsules are used, dosing based upon a strict milligram per kilogram paradigm is difficult given that those are manufactured only in a limited number of fixed-dose formulations. Therefore, pediatric dosing is typically based upon weight bands where, if not carefully studied, there could be significant differences in the milligram per kilogram exposure. Lastly, the task of drug exploration in pregnant women is shared by both obstetrical and pediatric investigators, as some ARVs have significant transplacental transfer and may be candidates for inhibiting mother-to-child HIV transmission.

\section{Darunavir PK clinical trial data}

Darunavir is FDA approved for pediatric populations 6-18 years of age and is available in $75,150,400$, and $600 \mathrm{mg}$ tablets. Dosing recommendation for adolescents $>40 \mathrm{~kg}$ is $600 \mathrm{mg}$ twice daily and DRV must be co-administered with RTV $100 \mathrm{mg}$. For ARV-naïve adolescents $>18$ years of age, DRV may be administered as $800 \mathrm{mg}$ once daily with RTV $100 \mathrm{mg}$. While once daily dosing for adolescents $<18$ years of age has not been studied, adherence considerations lead some pediatric HIV specialists to use this same once-a-day dose for adolescents $>40 \mathrm{~kg}$, even in instances where the adolescent was not ARV-naïve.

Long-term dosing in children 3-6 years of age has been studied in the ARIEL (dArunavir in tReatment experienced pEdiatric population) trial, a 48-week, open label, phase II study to evaluate DRV/r in children 3 to $\leq 6$ years of age of weights 10 to $\leq 20 \mathrm{~kg}$. Darunavir, available as a suspension of $100 \mathrm{mg} / \mathrm{mL}$, was initially administered at a dose of $20 \mathrm{mg} / \mathrm{kg}$ twice daily plus RTV $2.6-3.2 \mathrm{mg} / \mathrm{kg}$ twice daily which, after the initial PK evaluation, was changed to $25 \mathrm{mg} / \mathrm{kg}$ twice daily for participants between 10 to $\leq 15 \mathrm{~kg}$ and a $375 \mathrm{mg}$ twice daily for those 15 to $\leq 20 \mathrm{~kg}$. Those in this latter group were then given the option to switch to the tablet formulations. Ritonavir suspension was administered to the lower-weight group and the $50 \mathrm{mg}$ tablet was used in the higher-weight group. The specifics of the observed PK parameters have not been reported for this trial. At 24 weeks, $55 \%$ had achieved a HIV RNA $<50$ copies $/ \mathrm{mL}$, the mean CD4 increased 3.9\% (109 cells $\left./ \mathrm{mm}^{3}\right)$, and there were no new safety concerns compared with the established profile of DRV/r treatment. ${ }^{23}$ Darunavir will not be studied in children $<3$ years of age due to observed toxicity and mortality seen in juvenile rats who received doses ranging from 20 to $1000 \mathrm{mg} / \mathrm{kg}$.

Children and adolescents, 6-18 years of age and weighing $>20 \mathrm{~kg}$, were evaluated in the DELPHI trial. ${ }^{12}$ In the initial part of the study, participants were randomized to one of two weight band doses: Group A treated at a dose equivalent to the adult dose of DRV/r (600/100 mg twice daily) or, Group B, a dose approximately $20 \%-33 \%$ higher than the 
Group A. The Group A dose demonstrated an $\mathrm{AUC}_{24 \mathrm{~h}}$ of $81 \%$ and $\mathrm{C}_{0 \mathrm{~h}}$ of $91 \%$ of adult values while the Group B dose weight band dose of DRV 11-19 mg/kg and RTV 1.5-2.5 mg/ $\mathrm{kg}$ twice daily resulted in DRV exposure of $102 \%$ and $114 \%$ of the respective PK values in adults (see Figure 1, Tibotec pharmaceuticals data on file). The decision to go forward with the higher dose was based on both the AUC and $\mathrm{C}_{\text {min }}$ values as well as the finding that this higher dosing schema was well tolerated. This was confirmed in Stage II of this trial which has led to the Group B dose being granted regulatory approval for use in these age and weight groups. An important and practical limitation to this study was that, for participants $<40 \mathrm{~kg}$, the RTV was administered as the liquid preparation, which is associated with significant palatability issues leading to poor adherence. Clinically, most pediatric HIV experts will use the $100 \mathrm{mg}$ RTV tablet for anyone who can swallow a pill.

In adults, after twice daily oral administration of DRV $600 \mathrm{mg}$ with RTV $100 \mathrm{mg}$, bioavailability is $82 \%$, the $\mathrm{T}_{\max }$ is approximately $2.5-4$ hours with $95 \%$ of the agent bound to plasma proteins, primarily to plasma alpha 1-acid glycoprotein. In the liver, DRV primarily undergoes oxidative metabolism by CYP enzymes, predominantly CYP3A. The terminal elimination half-life of DRV is approximately 15 hours when co-administered with RTV. There may be an effect of different CYP3A polymorphisms on DRV/r PK which may be further defined over time. The reported DRV $\mathrm{AUC}_{12}$ and trough levels varied according to dosing frequency, dose, and co-administered therapy (ie, etravarine): for the $600 / 100 \mathrm{mg}$ twice daily dose, the $\mathrm{AUC}_{12}$ was approximately $11,500 \mathrm{ng}^{*} \mathrm{~h} /$ $\mathrm{mL}$ and the $\mathrm{C}_{0 \mathrm{~h}}$ was approximately $3400 \mathrm{ng} / \mathrm{mL}$. These values are not affected by concurrent hepatitis $\mathrm{B}$ or $\mathrm{C}$ infection in the presence of little or moderate hepatic function with little available information about dosing in the presence of severe liver dysfunction and treatment in this setting is not recommended. Similarly, in patients with mild to moderate renal impairment, there are no significant changes in DRV PK although there are limited data about these parameters in the presence of severe renal dysfunction. Lastly, DRV should be taken with food as it increases AUC and $\mathrm{C}_{\max }$ values by approximately $30 \%$ and requisite RTV administration is better tolerated when administered with food. ${ }^{24}$

\section{Specific PK issues related to ritonavir boosting}

Darunavir must be administered in conjunction with RTV as this latter agent inhibits CYP 3A4 and increases DRV exposure by approximately 14-fold, allowing therapeutic DRV exposure. Darunavir is metabolized by the cytochrome P450 3A4 and, in addition to being a substrate of this enzyme,

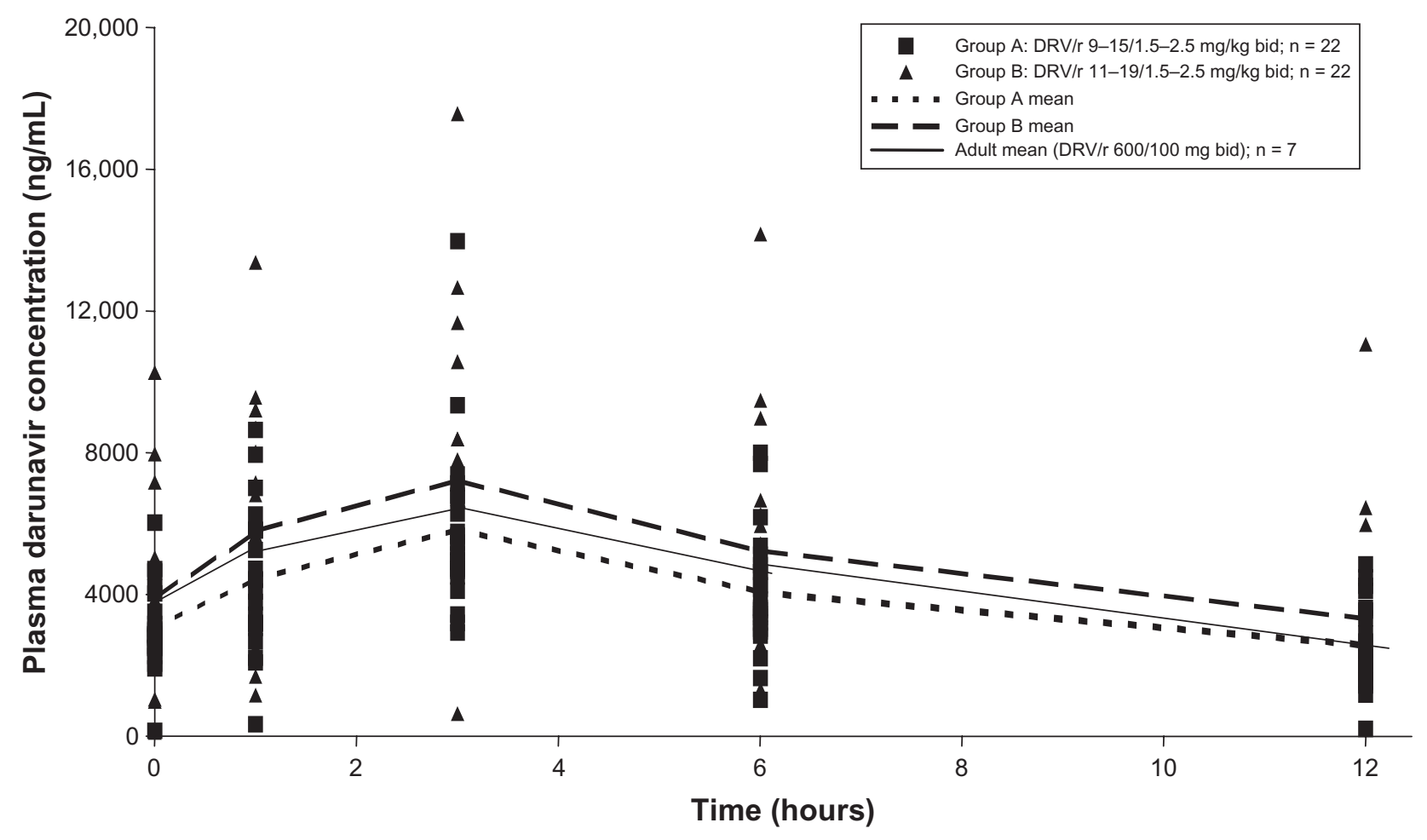

Figure I Low-dose ritonavir-boosted darunavir plasma concentrations in pediatric patients. Abbreviations: bid, twice daily; DRV/r, ritonavir-boosted darunavir. 
it also inhibits CYP 3A4 activity which may lead to altered metabolism of other medications, which may result in greater exposure to these drugs and added toxicity. Conversely, drugs that induce CYP3A activity would be expected to accelerate clearance of both DRV and RTV resulting in subtherapeutic levels of these two agents and decreased DRV efficacy. The interactions may be between DRV and RTV, or other drugs taken by the patient. Interactions affecting drug levels may have significant effects on peak and trough values which may influence efficacy and/or toxicity. These drug-drug interactions are complex and, over time, are being better defined. Clinicians are urged to remain vigilant about the potential for drug-drug interactions by exploring a number of on-line references such as http:/www.hivdruginteractions.org/ (University of Liverpool, UK), http:// hivinsite.ucsf.edu/insite? page=ar-00-02 (University of California, San Francisco), http://www.aidsinfo.nih.gov/ guidelines/(NIH treatment guidelines) and others.

\section{Implications for the future}

Important PK issues that need to be further addressed and reported include dosing parameters for children 3-6 years of age as well as use of this agent during pregnancy and its disposition in newborns born to infected women taking this DRV during pregnancy. Additionally, many of the common drug-drug interactions in children have not been well studied including DRV interactions with etravirine, a combination that is commonly used in infected adults. ${ }^{25}$ Of importance, as metabolism of these agents may vary across different pediatric developmental stages resulting in different levels of drug exposure, an important interaction may occur in one pediatric age group but not in another. Finally, raltegravir, a novel agent and class (integrase inhibitor) may provide added potency when combined with DRV/r. Moreover, a raltegravir chewable preparation is currently being investigated. ${ }^{26}$

\section{Darunavir resistance}

\section{Basic science: in vitro studies}

Darunavir was explicitly developed for the treatment of patients with highly PI-resistant HIV. Its flexible molecular structure facilitates the binding to and inhibition of both wild type protease (PR) as well as PR containing $>10-14$ PI resistance-associated mutations (RAMs). In vitro studies have demonstrated binding affinities for the wild-type and multi-drug resistant enzyme to be $2-3$ and 1.5 orders of magnitude higher than for first-generation PIs, respectively. ${ }^{27}$ These structure-function relationships demonstrate that DRV is a potent second-generation PI which could be used in treatment-experienced as well as antiretroviral-naïve patients

\section{$\mathrm{PI}$ resistance mutations and darunavir activity}

Darunavir exhibits an intrinsically high genetic barrier to resistance, making it exceedingly difficult to generate drugresistant mutants in tissue culture. Those mutations that have been selected in vitro do not confer resistance to DRV when introduced into HIV, either singly or in combination via site-directed mutagenesis, nor are these mutations observed in resistant clinical isolates. As such, most of the resistance data that are now published and have been incorporated into the IAS, Stanford, and European databases and guidelines are derived from the pivotal DRV registrational trials in treatment-experienced populations (DUET 1-2, POWER 1-3, ARTEMIS, and TITAN). This information is in constant flux and updated regularly, because as more DRV exposure occurs among HIV-infected patients, less common resistance mutations or mutational interactions will emerge, and their influence on DRV susceptibility examined.

Since formal clinical trials of this drug in the pediatric HIV-infected population are limited primarily to the DELPHI study, ${ }^{12}$ most of the resistance data and interpretations discussed in this section are extrapolated from the adult experience. To summarize the data in the simplest and perhaps most compelling manner, most highly treatment-experienced patients with extensive PI-exposure respond to DRV-based regimens if they have less than 10-14 PI RAMs. Moreover, most of the trials have proven that if at least two fully active drugs are incorporated in the regimen (etravirine and enfuvirtide in the DUET and POWER studies, respectively) the risk of treatment failure due to baseline resistance was significantly diminished. Of course, there are certain protease mutations that may negatively impact DRV susceptibility more than others; these mutations, listed in Table 1, form the basis for the DRV resistance profiles published in the IAS, Stanford, and other similar databases. Initial analyses of the POWER and DUET trial datasets demonstrated that the presence of $\geq 3$ DRV RAMs at baseline was predictive of higher rates of treatment failure. ${ }^{30}$ Notably, each of these DRV RAMs was present alongside a median number of 13-15 general PI RAMs, illustrating the heavy treatment experience of the study participants. More recent scrutiny of these datasets confirmed the negative and positive influence on the virologic response to DRV of PR mutations 147V, I54M, T74P, I84V, and V82A. ${ }^{31}$ Serendipitously, the beneficial V82A mutation occurs commonly in treatment experienced patients and can 
Table I Protease inhibitor resistance-associated mutations associated with darunavir, fos-amprenavir, and tipranavir

IAS (Dec 20I0) ${ }^{\mathrm{a}}$

VIII V32I L33F I47V I50V I54M/L T74P L76V I84V L89V

Stanford HIV Drug Resistance Database (Nov 2008) ${ }^{\mathrm{b}}$

VIII V32I L33F I47VIA I50V I54M/L G73S/T/C L76V

I84VIA/C L89V

Other:

V82F (potential cross-resistance)

V82A (linked to improved response to DRV/r in adults with multiple DRV RAMs)

Darunavir/Fos-amprenavir mutation overlap (IAS Dec 2010)

V32I I47V I50V I54M/L/V G73S L76V I84V

Tipranavir mutation profile (IAS Dec 2010)

LIOV L33F M36I/L/V K43T M46L I47V I54M/A/V Q58E H69K/

RT74P V82L/T N83D I84V L89V/I/M

Notes: Bolded positions indicate primary or major protease inbibitor mutations. ajohnson et $\mathrm{al}^{28}$; ${ }^{\mathrm{b}}$ Rhee SY et al. ${ }^{29}$

Abbreviations: DRV/r, ritonavir-boosted darunavir; RAM, resistance-associated mutations.

be selected as a primary or accessory mutation with all of the commercially available PIs except DRV.

As mentioned earlier, pediatric studies of DRV are limited, with predictable outcomes based on the potency and tolerability of this drug. When faced with a decision whether to use DRV as part of an early or late salvage regimen, clinicians would benefit from having some a priori knowledge about the probability of finding baseline DRV RAMs according to their patients' previous treatment histories and resistance tests. Such information would enable the clinician to accurately predict whether their patient would respond to a DRV-based regimen and would provide time to discuss various treatment options while definitive resistance testing results are pending. These data would be critical in settings where resistance testing is not available, either due to resource limitations or insurance issues. Previous resistance studies in adults have demonstrated that $>90 \%$ harbored $<3$ DRV RAMs, while about $67 \%$ were free of these mutations. The Collaborative HIV Pediatric Study (CHIP) has recently reported on the prevalence of DRV RAMs among a large group of PI-naïve $(\mathrm{N}=344 ; 93 \%$ nonsubtype $\mathrm{B})$ and $\mathrm{PI}$-experienced $(\mathrm{N}=156$; $86 \%$ nonsubtype B) HIV-infected children from the UK. ${ }^{32}$ Unless DRV-associated mutational patterns in non-B subtypes turn out to be significantly different from the profiles described with subtype B, their finding that DRV RAMs were rare among PI-naïve children (3\%) was not surprising. Furthermore, among PI-experienced patients, of which 35\% had received LPV/r as their only PI, only three (2\%) had three DRV RAMs. Factors associated with higher resistance to DRV included $>2$ years of PI exposure and greater levels of viremia. The authors concluded that DRV would be an appropriate PI to use in initial as well as salvage therapy situations. Further information on baseline DRV resistance among treatmentexperienced pediatric patients is currently being collected in the international ARIEL trial. ${ }^{23}$ Of 27 patients enrolled in the study ( $56 \%$ male, mean age 4.6 years at screening) and reported on at the 18th CROI 2011, there was a median of 0 primary PI mutations, 0 DRV RAMs, and four minor PI RAMs at screening. Robust virologic responses were noted at 24 weeks $(88 \%<400$ copies/mL, ITT-TLOVR), and no patients with paired baseline and 24 week isolates exhibited any new genotypic or phenotypic NRTI or PI resistance.

Despite the limited amount of baseline DRV resistance in multiple PI-experienced pediatric and adult patients, debate continues to linger about the role of fos-amprenavir exposure and subsequent DRV failures. As shown in Table 1, fosamprenavir and DRV RAMs share considerable overlap with each other. Although several studies testing DRV/r in highly ARV-experienced patient cohorts have reported a significant risk of treatment failure in patients harboring the specific fosamprenavir mutational profile (V32I + I47V or I50V), more critical examination of the data suggests otherwise and has led to the conclusion that previous fos-amprenavir exposure/ failure should not preclude the use of DRV in future salvage regimens. Finally, because of the lack of significant overlap between the mutation profiles for TPV and DRV (Table 1), patients who develop resistance to either of these PIs should be able to safely sequence to the other PI. However, it is important to note that TPV and DRV have considerably different toxicity profiles, and that TPV cannot be co-administered with etravirine due to an adverse drug interaction effect. ${ }^{33}$

\section{Conclusion}

Our center, located in an inner city public hospital, provides primary and specialized care to approximately $250 \mathrm{HIV}-$ infected children/adolescents. Most of the children we follow are perinatally infected, and as they reach adolescence and young adulthood, their management has become particularly challenging not only because of the inherently complex nature of adolescence itself but also because of the duration and progression of the infection and extensive drug resistance. In addition, we are entering an era when vertically infected adolescents and young adults are having children of their own, and transmission of multidrug-resistant HIV becomes a real risk between partners and mother to child. ${ }^{34}$ The recovery and preservation of immune function in this population depends on the availability of new effective drugs coupled with 
behavioral and psychosocial interventions aimed at optimizing adherence and keeping patients engaged and in care. Clinicians caring for these complex but still very young patients always need to balance the goals of achieving good short-term outcomes with the need to keep in mind the preservation of future options. Our clinical experience with DRV among the adolescent and young adult patients with multidrug-resistant HIV who attend our busy outpatient clinic is shaped by an evidence-based approach but tempered by the knowledge that complex psychosocial factors may sabotage a theoretically favorable outcome. Therefore we are occasionally forced to make unorthodox decisions to not only foster patient adherence but to achieve "buy in" that they need these medications in order to live. For example, in certain situations we have prescribed once daily DRV/r (800 mg/100 mg) in patients whose viruses harbor one or more DRV RAMs, knowing full well that the regimen may be insufficiently potent over the long term but honoring their unwavering request for a once daily regimen. Future studies on resistance and treatment outcomes should address this issue, which will become more relevant with the impending approval of several new once daily ARVs from the NNRTI and integrase inhibitor classes.

\section{Disclosure}

The authors declare no conflicts of interest in relation to this paper.

\section{References}

1. Gortmaker S, Hughes M, Cervia J, et al. Effect of combination therapy including protease inhibtors on mortality among children and adolescents infected with HIV-1. N Engl J Med. 2001; 345:1522-1528.

2. Gona P, van Dyke R, Williams PL, et al. Incidence of opportunistic and other infections in HIV-infected children in the HAART era. JAMA. 2006;296:292-3000.

3. Murphy DA, Sarr M, Durako SJ, Moscicki AB, Wilson CM, Muenz LR; Adolescent Medicine HIV/AIDS Research Network. Barriers to HAART adherence among human immunodeficiency virus-infected adolescents. Arch Pediatr Adolesc Med. 2003;157:249-255.

4. Merzel C, Vandervanter N, Irvine M. Adherence to antiretroviral therapy among older children and adolescents with HIV: a qualitative study of psychosocial contexts. AIDS Patient Care STDS. 2008;22: 977-987.

5. Kearns GL, Abdel-Rahman SM, Alander SW, Blowey, DL, Leeder JS, Kaufmann RE. Developmental pharmacology - drug disposition, action, and therapy in infants and children. $N$ Engl $J$ Med. 2003;349:1157-1167.

6. Fletcher CV. Antiviral drug-drug interaction considerations for HIVinfected children. Pediatr Infect Dis J. 2009;28:429-430.

7. Menson EN, Walker AS, Sharland N, et al; Collaborative HIV Paediatric Study Steering Committee. Underdosing of antiretrovirals in UK and Irish children with HIV as an example of problems in prescribing medicines to children, 1997-2005: cohort study. BMJ. 2006;332:1183-1187.

8. Floren LC, Wiznia A, Hayashi S, et al; Pediatric AIDS Clinical Trials Group Protocol 377 Team. Nelfinavir pharmacokinetics in stable human immunodeficiency virus-positive children: Pediatric AIDS Clinical Trial Group Protocol 377. Pediatrics. 2003;112:e220-e227.
9. Panel on Antiretroviral Therapy and Medical Management of HIV-infected Children. Guidelines for the Use of Antiretroviral Agents in Pediatric HIV Infection: August 11, 2011. Rockville, MD: AIDSinfo; 2011:3-7. Available from: http://aidsinfo.nih.gov/ContentFiles/PediatricGuidelines.pdf. Accessed September 5, 2011.

10. Clotet B, Bellos N, Molina JM, et al; POWER 1 and 2 study group. Efficacy and safety of darunavir-ritonavir at week 48 in treatment experienced patients with HIV-infection in POWER 1 and 2: a pooled subgroup analysis of data from two randomised trials. Lancet. 2007;369:1169-1178.

11. Ortiz R, Dejesus E, Khanlou H, et al. Efficacy and safety of once daily darunavir/ritonavir versus lopinavir/ritonavir in treatment-naïve HIV-1-infected patients at week 48. AIDS. 2008;22:1389-1397.

12. Blanche S, Bologna R, Cahn P, et al. Pharmacokinetics, safety and efficacy of darunavir/ritonavir in treatment experienced children and adolescents. AIDS. 2009;23:2005-2013.

13. Rosenberg M, Abadi J, Dobroszycki J, Wiznia A. Progress and challenges in antiretroviral therapy of HIV-infected children. Curr Infect Dis Rep. 2006;8:324-331.

14. Volberding PA, Deeks SG. ARV therapy and management of HIV infection. Lancet 2010;376:49-52.

15. McKeage K, Scott LJ. Darunavir in treatment-experienced pediatric patients with HIV-1 infection. Paediatr Drugs. 2010;12:123-131.

16. Neely M, Kovacs A. Managing treatment-experienced pediatric and adolescent HIV patients: role of darunavir. Ther Clin Risk Manag. 2009;5:595-615.

17. Valdez J, Daniel Berger D, McMurchie M. Efficacy and safety of darunavir-ritonavir compared with that of lopinavir-ritonavir at 48 weeks in treatment-experienced, HIV-infected patients in TITAN: a randomised controlled phase III trial. Lancet. 2007;370:49-58.

18. Thuret I, Chaix M, Tamalet C, et al. Raltegravir, etravirine and r-darunavir combination in adolescents with multidrug-resistant virus. AIDS. 2009;23:2364-2366.

19. Arribas JR, Horban A, Gerstoft J, et al. The MONET trial: darunavir/ ritonavir with or without nucleoside analogues, for patients with HIV RNA below 50 copies/ml. AIDS. 2010;24:223-230

20. De Meyer SMJ, Spinosa-Guzman S, Vangeneugden TJ, de Béthune MP, Mirales GD. Efficacy of once-daily darunavir/ritonavir 800/100 mg in HIV-infected, treatment-experienced patients with no baseline resistance-associated mutations to darunavir. J Acquir Immune Defic Syndr. 2008;49:179-182.

21. Mills AM, Nelson M, Jayaweera D, et al. Once-daily darunavir/ritonavir vs lopinavir/ritonavir in treatment-naive, HIV-1-infected patients: 96-week analysis. AIDS. 2009;23:1679-1688.

22. Delaugerre C, Buyck JF, Peytavin G, et al. Factors predictive of successful darunavir/ritonavir-based therapy in highly antiretroviralexperienced HIV-1-infected patients the DARWEST study. J Clin Virol. 2010;47:248-252.

23. Violari A, Bologna R, Kimutar R, et al. ARIEL: 24-week safety and efficacy of DRV/r in treatment-experienced 3- to <6-year-old patients. Poster 713, 18th CROI 2011 Feb 27-Mar 2, Boston, USA.

24. Prezista ${ }^{\circledR}$ Full Prescribing Information. Tibotec Therapeutics, Raritan, NJ. Revised, December 2010.

25. Katlama C, Haubrich R, Lalezari J, et al. Efficacy and safety of etravirine in treatment-experienced, HIV-1 patients: Pooled week 48 analysis of two randomized, controlled trials. AIDS. 2009;23:2289-2300.

26. Wiznia A, Samsom P, Acosta E, et al. Safety and efficacy of raltegravir on pediatric HIV infection. Preliminary analysis from IMPAACT P1066. Poster 874, 16th CROI 2009 Feb 8-11, Montreal, Canada.

27. Lefebvre E, Schiffer CA. Resilience to resistance of HIV-1 protease inhibitors: profile of darunavir. AIDS Rev. 2008;10:131-142.

28. Johnson VA, Brun-Vézinet F, Clotet B, et al. Update of the drug resistance mutations in HIV-1: December 2010. Top HIV Med. 2010;18:156-163.

29. Rhee SY, Gonzales MJ, Kantor R, Betts BJ, Ravela J, Shafer RW. Human immunodeficiency virus reverse transcriptase and protease sequence database. Nucleic Acids Res. 2003;31:298-303. 
30. De Meyer S, Dierynck I, Lathouwers E, et al. Phenotypic and genotypic determinants of resistance to darunavir: analysis of data from treatmentexperienced patients in POWER 1, 2, 3 and DUET-1 and 2. Antiviral Ther. 2008;13:Suppl 3:A33.

31. De Meyer S, Descamps D, Van Baelen B, et al. Confirmation of the negative impact of protease mutations I47V, I54M, T74P, I84V and the positive impact of protease mutation V82A on virological response to darunavir/ritonavir. Antiviral Ther. 2009;14:Suppl 1:A147.
32. Boyd K, Walker S, Dunn D, et al. The prevalence of darunavir associated mutations in PI-naive and PI-experienced HIV-1 infected children in the UK. Poster 851, 17th CROI 2010 Feb 16-19, San Francisco, USA.

33. Tremblay CL. Combating HIV resistance - focus on darunavir. Ther Clin Risk Manag. 2008;4:759-765.

34. Phillips UK, Rosenberg MG, Dobroszycki J, et al. Pregnancy in women with perinatally acquired HIV-infection: outcomes and challenges. AIDS Care. 2011;11:1-7.

\section{Publish your work in this journal}

Adolescent Health, Medicine and Therapeutics is an international, peer-reviewed, open access journal focusing on health, pathology, and treatment issues specific to the adolescent age group. All aspects of health maintenance, preventative measures and disease treatment interventions are addressed within the journal and practitioners from all disciplines are invited to submit their work as well as healthcare researchers and patient support groups.. The manuscript management system is completely online and includes a very quick and fair peerreview system. Visit http://www.dovepress.com/testimonials.php to read real quotes from published authors.

Submit your manuscript here: http://www.dovepress.com/adolescent-health-medicine-and-therapeutics-journal 